\title{
Adaptive Forward Error Correction for Best Effort Wireless Sensor Networks
}

\author{
Kan Yu*, Filip Barac ${ }^{\dagger}$, Mikael Gidlund ${ }^{\ddagger}$ and Johan Åkerberg*‡ \\ ${ }^{*}$ Mälardalen University, Sweden \\ ${ }^{\dagger}$ Mid Sweden University, Sweden \\ $\ddagger$ ABB AB, Corporate Research, Sweden
}

\begin{abstract}
In this work we propose an Adaptive Forward Error Correction (AFEC) algorithm for best effort Wireless Sensor Networks. The switching model is described in terms of a finitestate Markov model and it is based on the channel behavior, observed via Packet Delivery Ratio in the recent past. We compare the performance of AFEC with static FEC, as well as uncoded transmissions. The results demonstrate a gain in PDR achieved by introducing FEC coding in uncoded IEEE 802.15.4 transmissions, as well as the advantages over static FEC schemes, namely increased throughput and reduced energy consumption. The proposed solution is IEEE 802.15.4-compliant and requires no additional feedback channels.
\end{abstract}

\section{INTRODUCTION}

Cost reduction and increased flexibility are the principal reasons for introducing Wireless Sensor Networks (WSNs) into a plethora of applications, ranging from consumer market utilities to environmental monitoring. WSN deployment sites frequently abound with non-line-of-sight (NLOS) communication, electromagnetic disturbances and moving objects. $\mathrm{Nu}-$ merous field studies show the temporal variations of Received Signal Strength Indicator (RSSI) on both large and small timescale that may amount up to $25 \mathrm{~dB}$ [1].

IEEE 802.15.4 standard, targeting low-power low-rate radios, does not provide any advanced error-control mechanisms [2]. Instead, it combines error detection by Cyclic Redundancy Check (CRC) with Automatic Repeat Request (ARQ). This concept not only lacks proactive means for error correction, but it also results in increased communication latency. Another approach to improving transmission reliability is Forward Error Correction (FEC), based on recovering from errors by adding redundancy to the original data. Although additional redundancy reduces transmission efficiency, FEC is still a more preferable solution, because it may improve both reliability and latency.

The amount of redundancy and error correction capabilities of FEC codes are directly proportional, and the choice of FEC code has a direct influence on system throughput and energy consumption. Although the high reliability can be achieved by using a FEC code with the strongest error correction capability, the common practice of static "design for the worst case scenario" can, in terms of FEC codes, quite often be an overkill. In other words, due to variations of wireless channel conditions, it might not be necessary to employ the strongest FEC code all the time. On the other hand, applications are usually designed to tolerate a certain amount of packet loss, so it is acceptable to choose a less strong FEC code to fulfill the application requirement. A possible drawback of static FEC schemes is that they may fail to fulfill the PDR requirement (in case the code is not strong enough) or they may demonstrate low throughput at an additional energy cost for transmitting the redundancy. Therefore, a dynamic selection of FEC coding may contribute to a wiser exploitation of communication resources.

Extensive research has been done on adaptive FEC in WSNs. In [3], the authors select one out of several possible BCH codes for Adaptive Data Length-Forward Error Correction, based on the fraction of successfully received packets. However, their scheme requires additional feedback channels. Introduction of another feedback channel (in addition to already existing ACK feedbacks) would violate the IEEE 802.15.4 standard. A Hybrid-ARQ-Adaptive-FEC scheme in [4] is based on collecting Signal-to-Noise Ratio (SNR) information. In environments where variations of RSSI are frequent and intensive, observation of short-term variations of SNR can fail in providing the information about the overall trend of change of propagation conditions. In [5] and [6] adaptive FEC methods aiming for low power consumption are proposed, but these solutions are based on multi-path transmissions, which are highly dependent on the network topology. Thus, significant delays in case of transmission errors may arise.

We propose an Adaptive Forward Error Correction (AFEC) coding scheme on the Medium Access Control (MAC) layer. This work is the first step towards our final solution. It reveals the benefits and trade-offs coupled with AFEC concept, as well as obstacles that could be expected towards reaching the finite form. Starting from a set of codes with different error correcting capabilities, we define an adaptive algorithm for switching between them, based on a finite-state Markov model, where each code is assigned a state. We define a switching criterion based on the number of acknowledgements (ACKs) received inside a window of $L$ previously transmitted packets. Packet Error Rate (PER) within the window is calculated and compared with a predefined switching threshold $P E R_{T}$ to determine whether to switch to a code with higher or lower error-correcting capability. The coding introduced by AFEC is IEEE 802.15.4-compliant and the adaptive algorithm do not require a dedicated feedback channel to obtain channel state information, since AFEC evaluates the channel from ACKs, which are already defined in this standard. The goal of 
AFEC is to maintain a Packet Delivery Ratio (PDR) required by the application in an adaptive manner. By adjusting the amount of redundancy to the current channel state, the energy is consumed more rationally. We evaluate the performance of AFEC against the fixed coding approach on the Rayleigh channel model and investigate the relationships between the performance and switching thresholds and strategies. The gains with respect to uncoded IEEE 802.15.4 transmissions are also considered. Simulation results show that, while achieving the required PDR, the proposed solution can reduce the energy consumption by transmitting fewer packets and obtain higher throughput than static FEC schemes.

In Section II the AFEC scheme is proposed based on Markov model in the MAC layer. Section III describes the simulation setup and Section IV presents and discusses the results. Conclusions are summarized in Section V.

\section{Proposed Adaptive FEC Scheme for IEEE 802.15.4 BASED ON MARKOV MODEL}

In this section, we describe the MAC layer FEC scheme and propose our adaptive FEC strategy. Code adaptation is based on the channel quality estimates. The estimation is executed on MAC layer, because the purpose of transmission is to deliver MAC frames.

\section{A. Proposed Adaptive FEC Coding Scheme on MAC layer}

In [7], a MAC layer FEC scheme was proposed and its flexibility and compatibility with IEEE 802.15.4 were shown. However, this FEC scheme can be further optimized. A FECcoded data frame is shown in Figure 1. After the header and original payload are encoded, the redundant data is stored separately in a special $l$-byte FEC field at the end of the payload. This field contains the version of FEC code used to encode the packet. Each node has all available FEC codes implemented, and chooses the decoding scheme based on the version number from the incoming packet. The FEC version field is excluded from the data FEC protection range, but it is encoded by a specific FEC code, known to all sensor nodes. Once a sensor node receives a packet, it will first calculate the offset of the FEC version field and decode it to find out which FEC code it should use to decode the remainder of the packet.

The advantage of this FEC scheme is that the feedback channel for indicating switching between FEC codes is no longer necessary - the packet itself contains the FEC version and guarantees the use of an appropriate FEC code, provided that the receiver is able to correctly decode the FEC version field. Another important benefit of AFEC is its flexibility for multi-hop networks. In a multi-hop transmission, the FEC code may be changed at each hop, if necessary. An intermediate sensor node performs error check in the packet by examining the Frame Control Sequence field. If this check shows that packet is uncorrupted, the node can directly extract the original data by removing the redundancy (which is placed at the end of the payload) without decoding the whole packet. Then this intermediate node can encode the packet by another FEC code

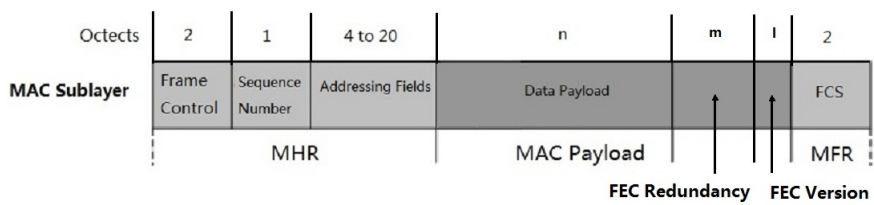

Fig. 1. Applying FEC scheme at the MAC layer

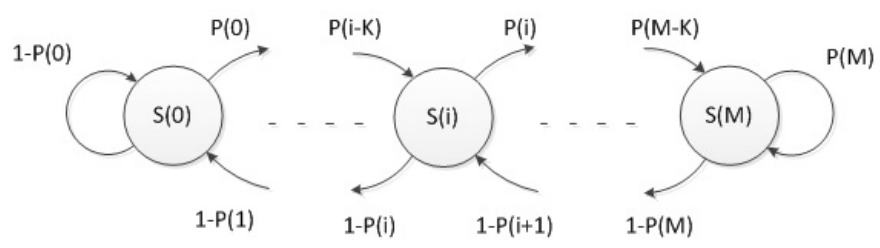

Fig. 2. Markov Model for the proposed scheme

and forward it. Hence, the workload for the routing nodes is reduced, which leads to smaller energy consumption and lower in-node processing time.

\section{B. Proposed FEC Switching Scheme Based on Markov Model}

The reliability is increased by our proposed solution from two aspects. The first feature is the very introduction of FEC coding, which is not defined by the original IEEE 802.15.4 standard. In addition, reconfiguration of FEC is introduced, in order to adapt to channel conditions and send just the required amount of redundancy. The aim of the proposed AFEC scheme is to maintain the PDR above the application requirement according to a predefined switching threshold $P E R_{T}$ by adaptively switching between codes of different correcting powers. The switching can be represented by Markov states. In each Markov state, a specific RS code is applied on the outgoing packets. This Markov model with $M$ states is illustrated in Figure 2, where $S(i)$ represents the state $i$. The transmission should start from state $S(0)$, i.e. the lightest FEC code, whereas $S(M)$ is the state with the strongest FEC code. $P(i), i<M$ indicates the transition probability from state $i$ to state $i+K$, where $K$ is an integer that indicates the jump step. The transition probability from state $i$ to state $i-1$ is $1-P(i)$, while $P(M)$ is the probability of staying in state $M$.

The adaptive switching concept works as follows: sender will evaluate the channel conditions and change the coding accordingly. In case of channel improvement, the receiver will switch to a less powerful FEC code. In case the channel has deteriorated, the transmitter will transit to a more powerful code.

The switching threshold $P E R_{T}$ has a corresponding PDR: $P D R_{T}=1-P E R_{T}$. In order to maintain this PDR, a node shall observe the incoming ACKs for $L$ previous packets that it has sent. Hence, only the history that can fit in a timewindow of certain length is considered. There are two reasons for this: firstly, if the entire history was considered, even very long bursts of packet errors would have a small impact on the total PER estimate, making the estimate rigid, outdated and inaccurate. Secondly, channel variations are intensive, so only a recent short history is relevant for the estimate. 
Furthermore, if wireless channel conditions change frequently, it is necessary to quickly adjust the coding and $L$ should be rather small. We define a packet window of length $L$ and observe the ratio of the number of transmitted packets and the received ACKs within this window as:

$$
P E R_{w i n}=1-\frac{N_{a c k}}{L}
$$

where $N_{a c k}$ is the number of received ACKs within the window and $P E R_{\text {win }}$ represents the PER within the window.

The window stretches over $L$ packets and it is shifted periodically - the time between two window shifts makes up one cycle. A window shift occurs after the waiting times for all $L$ acknowledgements expire. Since the shift is not incremental, each packet resides inside the window exactly once, and this time of residence equals one cycle. The algorithm of AFEC coding scheme at the transmitter side is described below.

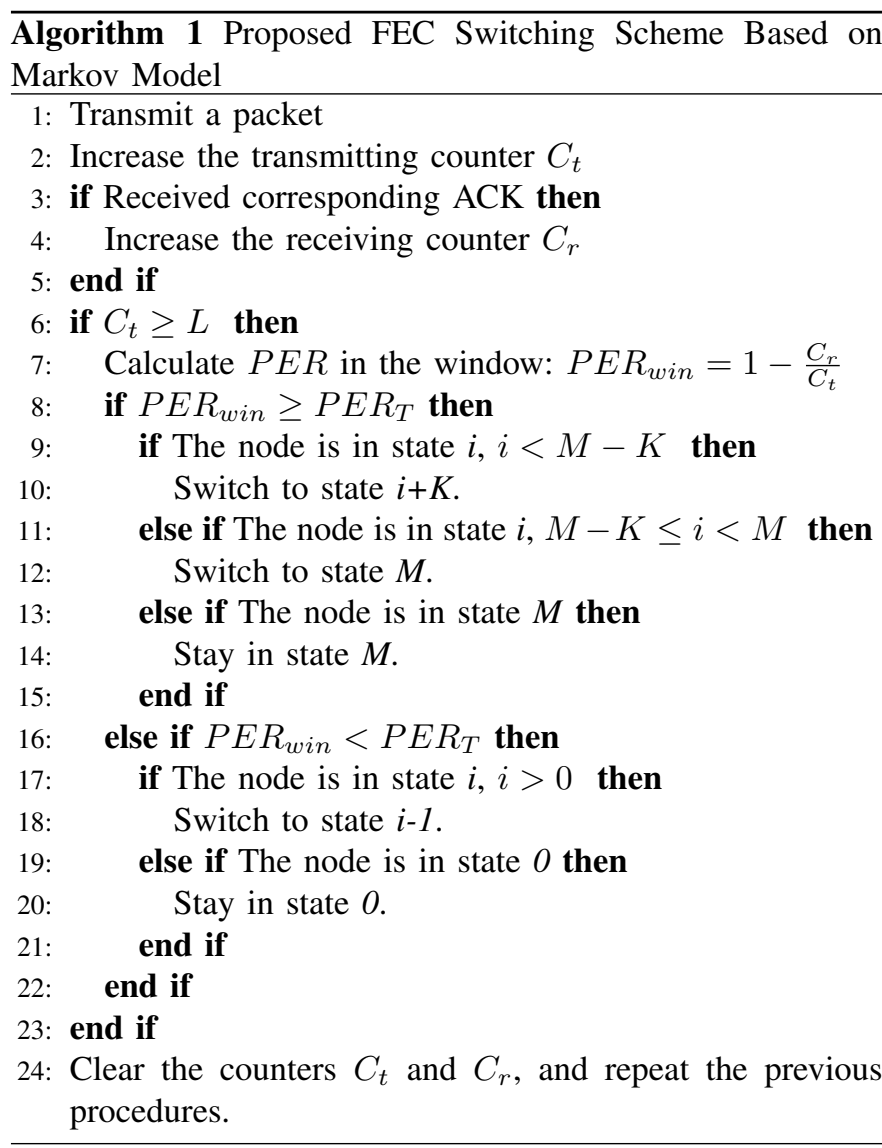

The code switching can be represented by a Markov chain with the states corresponding to the codes used and the equilibrium equations are:

$$
\begin{aligned}
& \pi_{0}=\left(1-P_{0}\right) \pi_{0}+\left(1-P_{1}\right) \pi_{1} \\
& \pi_{i}=\left(1-P_{i+1}\right) \pi_{i+1}, 0<i<K \\
& \pi_{i}=P_{i-K} \pi_{i-K}+\left(1-P_{i+1}\right) \pi_{i+1}, K \leq i \leq M \\
& \pi_{M}=P_{M-K} \pi_{M-K}+P_{M} \pi_{M} \\
& \sum_{i=0}^{M} \pi_{i}=1
\end{aligned}
$$

where $\left\{\pi_{i}: i=0,1, \ldots, M\right\}$ is the set of stationary probabilities.

According to our proposed scheme, switching to a stronger code occurs when the $P E R_{\text {win }}$ is larger than switching threshold $P E R_{T}$ with the transition probability $P_{i}$. Therefore, $P_{i}$ can be defined as:

$$
P_{i}=\operatorname{Pr}\left\{P_{e}(i) \geq P E R_{T}\right\}
$$

where $P_{e}(i)$ is the theoretical encoded PER in state $i$.

If we consider the Additive White Gaussian Noise (AWGN) channel model, BER $P_{b}$ without considering the phase noise is given by [8]:

$$
P_{b}=\frac{M_{s} / 2}{M_{s}-1} \sum_{k=1}^{M_{s}-1} \frac{(-1)^{k+1}}{k+1}\left(\begin{array}{c}
M_{s}-1 \\
k
\end{array}\right) e^{-\frac{k}{1+k} \frac{K_{b} E_{b}}{N_{0}}}
$$

where $K_{b}=\log _{2} M_{s}$ denotes the number of bits in one symbol, $M_{s}=16$ is the size of the signal constellation, and $E_{b}$ represents the energy per bit.

For the Rayleigh fading channel model, BER $P_{b}$ without considering the phase noise is given by [8]:

$$
P_{b}=\frac{M_{s} / 2}{M_{s}-1} \sum_{k=1}^{M_{s}-1}\left(\begin{array}{c}
M_{s}-1 \\
k
\end{array}\right) \frac{(-1)^{k+1}}{1+k+2 k K_{b} \sigma_{r}^{2} E_{b} / N_{0}}
$$

where $\sigma_{r}^{2}$ is a parameter characterizing the quasi-stationary Rayleigh flat-fading channel.

The $R S(n, k)$ code are used in AFEC scheme, and the probability that a codeword is wrongly decoded is given by [8]:

$$
P_{r}\left(P_{\text {sym }}, n\right)=\sum_{i=t+1}^{n}\left(\begin{array}{l}
n \\
i
\end{array}\right)\left(P_{\text {sym }}\right)^{i}\left(1-P_{\text {sym }}\right)^{n-i}
$$

where $n$ is the encoded packet length, $k$ is the original packet length, $t=(n-k) / 2$ and $P_{\text {sym }}$ is the symbol error probability of RS code, which can be calculated as:

$$
P_{\text {sym }}\left(P_{b}, l\right)=1-\left(1-P_{b}\right)^{l}
$$

In Equation 7, $l$ is the number of bits in an RS-coded symbol and $P_{b}$ can be found from (4) or (5).

If we consider an $N$-bit encoded packet, then the encoded $P E R, P_{e}(i)$ in (3) is:

$$
P_{e}(i)=1-\left(1-P_{\text {sym }}\right)^{\left\lceil\frac{N}{l}\right\rceil}
$$




\section{Selection of FEC codes}

FEC codes applied in WSNs should be lightweight in terms of memory footprint and execution time, due to the timing requirement for acknowledgements. IEEE 802.15.4 prescribes that a sender should wait for an acknowledgement for at most macAckWaitDuration symbols. Otherwise, the transmission is considered to have failed. Consequently, the receiver must decode an incoming packet within at most macAckWaitDuration symbols time. According to the IEEE 802.15.4 specification [9], the value of macAckWaitDuration can be calculated as $0.864 \mathrm{~ms}$ and the decoding time should be less than this value, in order to comply with the standard. According to the evaluation from [7], Reed-Solomon RS $(15,11)$ code meets this timing requirement. We select four additional RS codes, namely $\mathrm{RS}(15,13), \mathrm{RS}(15,9), \mathrm{RS}(15,7)$ and $\mathrm{RS}(15,5)$, whose complexity is in the order of $\mathrm{RS}(15,11)$ code. The differences between the performance of different codes shall be discussed in Subsection III-C.

\section{The choice of link quality metric}

The reliability of transmissions is quantified by the PDR. For this reason, PDR is chosen as the link quality metric, rather than immediate channel quality indicators such as RSSI and SINR. Although RSSI and SINR are easily deducible from incoming packets, they are not used as the channel quality indicators for several reasons. Numerous empirical studies have proven their high variability [1]. Typical wireless channel coherence times are much lower than the typical refresh rates and one realization of a random process with high variance does not provide enough information about the channel state. Using averaged RSSI or SINR as a metric would raise an additional issue of mapping the obtained values to different codes. Extracting RSSI or SINR from ACK packets is not appropriate because the wireless channel is asymmetric. Finally, high value of RSSI due to interference may provide wrong information about the channel state.

\section{E. The choice of window length}

For a given window length $L$, the set of possible values of $P E R_{c}$ spans from 0 to 1 with the step size of $\frac{1}{L}$. For small values of $L$, the granularity of channel estimates is very coarse. When the channel is estimated on a larger set of ACKs, the switching process becomes more stable and robust. If the window length is too large, the outdated history will result in an imprecise estimation of the current channel condition. For the experiments, we choose the window length of $L=20$, which gives a good resolution of measured PER.

\section{EXPERIMENTAL SETUP}

QualNet discrete event simulator [10] is used in the evaluations. It is a packet-level simulator and for this purpose it was extended to the bit-level. The details of this modification are given in Subsection III-A.
TABLE I

THE SIMULATION PARAMETERS

\begin{tabular}{cc}
\hline Parameter & Description \\
\hline Application layer & CBR application \\
MAC layer & IEEE 802.15 .4 without CSMA/CA and ARQ \\
Physical layer & IEEE 802.15 .4 with OQPSK and DSSS \\
Channel Model & Street Microcell [11] with Rayleigh fading \\
Packet size & 126 bytes +6 bytes preamble \\
Transmission power & $3 \mathrm{dBm}$ \\
\hline
\end{tabular}

\section{A. Propagation Environment}

The propagation environment is represented by the Rayleigh fading channel model [8]. The RSSI of a signal under Rayleigh fading exhibits fast variations, due to multipath propagation, non-line-of-sight communication and relative motion of transmitter, receiver and surrounding objects. Our channel model behaves similarly to the one observed in [1].

An empirical investigation of an IEEE 802.15.4 transmission error trace in [12] shows that $99 \%$ of bit errors in packets come in bursts of four or less. Jeong et al. [13] show that single- and double-bit errors dominate the error trace. Accordingly, the packet error process in this work is simulated by applying bursts of 1-4 bit errors, randomly distributed across the packet. The process of generating bit errors is described below:

- Rayleigh fading model of the simulator employs precomputed time series data sequence with different sample intervals, and captures the effect of Rayleigh fading in the resulting SINR of a packet.

- The resultant SINR is transformed into $B E R_{O Q P S K}$ by using BER table for OQPSK modulation.

- The corresponding number of corrupted bits, $n_{c o r r}$, equals: $n_{c o r r}=l \times B E R_{O Q P S K}$, where $l$ is the packet length in bits. These bit errors are randomly dispersed around the packet in bursts of one, two, three or four.

\section{B. Packet Reception Model}

An IEEE 802.15.4 packet starts with six bytes of preamble, packet delimiter and PHY layer header. This part of the packet is left uncoded, since FEC coding is executed on the MAC layer. Although FEC code is able to eliminate a certain number of errors, an irreparable packet error occurs if there exist bit errors in the preamble, irrespective of whether the remainder of the packet can be recovered or not.

\section{Code ranking}

The adaptive FEC algorithm is based on switching between FEC codes of different powers. Hence, it is necessary to establish a hierarchy among the codes, in order to provide a meaningful switching strategy. The code rates and error correction capabilities of all candidates are summarized in Table II. The code ranking has been experimentally confirmed, but the results are not presented due to spatial constraints.

From Table II, the code with the highest code rate will insert the least overhead into encoded packets, resulting in highest 
TABLE II

CODE RANKING IN TERMS OF ERROR CORRECTING POWER

\begin{tabular}{l|l|l}
\hline FEC code & Code Rate & $\begin{array}{l}\text { Error Correction } \\
\text { Capability [Sym] }\end{array}$ \\
\hline \hline 1. RS(15,5) & 0.33 & 5 \\
2. RS(15,7) & 0.467 & 4 \\
3. RS $(15,9)$ & 0.6 & 3 \\
4. RS $(15,11)$ & 0.733 & 2 \\
5. RS(15,13) & 0.867 & 1 \\
\hline
\end{tabular}

capacity for information payload. For $R S(n, k)$ code, the error correction capability is defined as $t=\lfloor(n-k) / 2\rfloor$, where $n$ and $k$ are the total number of symbols and the number of information symbols in a codeword.

\section{Simulation Results}

The scenarios are executed on a point-to-point topology. Two nodes, placed 50 meters apart, communicate over a Rayleigh fading channel. The transmitter is instructed to send at a refresh rate of $500 \mathrm{~ms}$.

This experiment is structured as follows: the transmitter is assigned 90,000 bytes of original data to be delivered to the receiver. Since encoded packet length is fixed and code rates differ, the amount of useful information in every packet depends on the code used. This will affect the time necessary to deliver all useful information. We consider three switching strategies, namely $U_{1} D_{1}, U_{2} D_{1}$ and $U_{3} D_{1}$, and compare them to static schemes. In the notation, $i$ and $j$ in $U_{i} D_{j}$ refer to upward and downward jumps between the codes in terms of number of ranks.

Figure 3 shows the way that AFEC balances between the higher robustness of strong codes and higher throughput of weaker codes. Each PER value for a fixed SNR is averaged over three transmissions of 90,000 bytes. Window length used is $L=20$ and $U_{3} D_{1}$ switching threshold is $P E R_{T}=0.15$. The PER values for AFEC are confined between the two strongest and three weakest codes, throughout most of the observed SNR range. The selected switching threshold for $U_{3} D_{1}$ is moderately high and the AFEC curve may be scaled up or down by setting the threshold higher or lower. The curve for an uncoded system is not shown, due to spatial constraints. However, uncoded transmissions have exhibited binary behavior: PER equals 1 up to $6 \mathrm{~dB}$ of SNR, after which it sharply drops to zero. This is understandable, considering the previously explained packet reception model - a single symbol error in the packet makes it permanently lost. Since the IEEE 802.15.4 standard prescribes no channel coding, the sole introduction of FEC produces a gain in reliability and throughput, as shown in Table III.

Table III shows simulation results for various switching thresholds and strategies under Rayleigh fading. The three strategies are tested with switching thresholds of $0,0.15$ and 0.25 , respectively. The window length used is $L=20$ packets. According to the results, $U_{3} D_{1}$ always provides the lowest PER among the strategies. The difference between strategies is the reaction in case the recent PER exceeds the switching

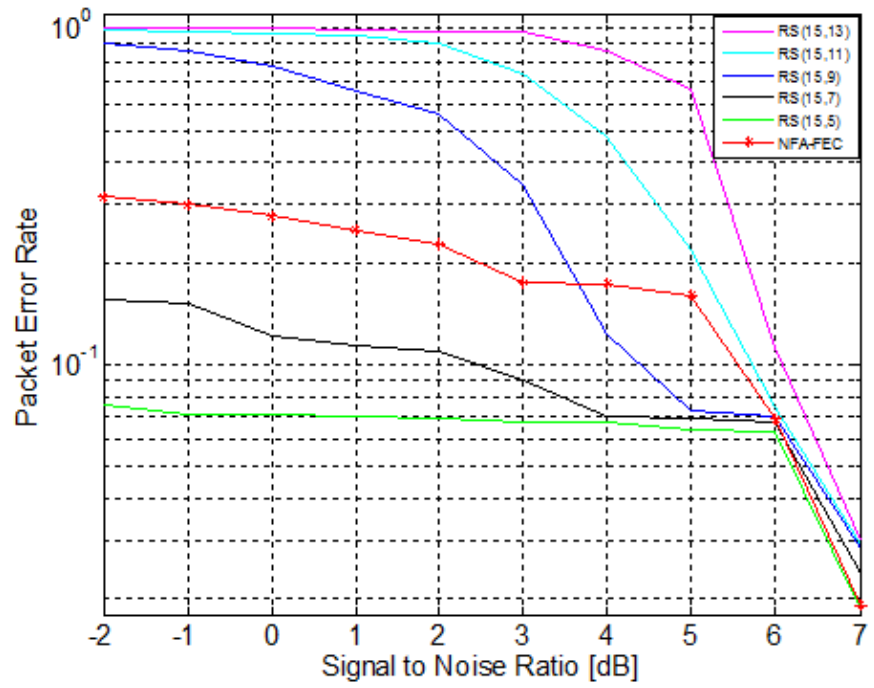

Fig. 3. Packet Error Rate of AFEC and static schemes for fixed SNR values

TABLE III

PERFORMANCE OF SWITCHING STRATEGIES, STATIC FEC AND AN UNCODED SYSTEM UNDER RAYLEIGH FADING

\begin{tabular}{l|l|l|l|l}
\hline Strategy & $\begin{array}{l}\text { Switching } \\
\text { threshold }\end{array}$ & $P E R$ & Throughput & $\begin{array}{l}\text { Number of } \\
\text { transmissions }\end{array}$ \\
\hline \hline$U_{1} D_{1}$ & 0.25 & $27.2 \%$ & $1,106 \mathrm{bits} / \mathrm{s}$ & 940 \\
$U_{2} D_{1}$ & 0.25 & $19.6 \%$ & $946 \mathrm{bits} / \mathrm{s}$ & 1,185 \\
$U_{3} D_{1}$ & 0.25 & $18.4 \%$ & $894 \mathrm{bits} / \mathrm{s}$ & 1,263 \\
\hline$U_{1} D_{1}$ & 0.15 & $17.4 \%$ & $924 \mathrm{bits} / \mathrm{s}$ & 1,264 \\
$U_{2} D_{1}$ & 0.15 & $14.2 \%$ & $866 \mathrm{bits} / \mathrm{s}$ & 1,387 \\
$U_{3} D_{1}$ & 0.15 & $13.3 \%$ & $804 \mathrm{bits} / \mathrm{s}$ & 1,505 \\
\hline$U_{1} D_{1}$ & 0 & $7.7 \%$ & $610 \mathrm{bits} / \mathrm{s}$ & 2,169 \\
$U_{2} D_{1}$ & 0 & $7.7 \%$ & $595 \mathrm{bits} / \mathrm{s}$ & 2,212 \\
$U_{3} D_{1}$ & 0 & $7.4 \%$ & $578 \mathrm{bits} / \mathrm{s}$ & 2,299 \\
\hline \hline Static RS(15,5) & none & $3.3 \%$ & $535 \mathrm{bits} / \mathrm{s}$ & 2,498 \\
Uncoded system & none & $43 \%$ & $332 \mathrm{bits} / \mathrm{s}$ & 2,498 \\
\hline
\end{tabular}

threshold. Strategy $U_{3} D_{1}$ has the highest jump, as it shifts three steps towards a stronger FEC state. The results of $U_{1} D_{1}$ show that when the channel conditions suddenly deteriorate, jumping one step towards a stronger FEC code may not be enough to overcome errors, which results in a worse reliability performance than jumping more steps. The PER of $U_{1} D_{1}$ is always higher than for other two strategies. Conversely, $U_{1} D_{1}$ provides the highest throughput out of three strategies. Similarly to $U_{2} D_{1}$ and $U_{3} D_{1}, U_{1} D_{1}$ jumps to a stronger code when necessary, but it makes a minimal jump. Hence, it is always biased towards weaker codes. A higher throughput, compared to other strategies, is a direct consequence of this bias, since weaker codes have higher code rate and more capacity remains for information payload.

The switching threshold directly influences the extent to which each of the codes will be used. Lower values of switching threshold $P E R_{T}$ imply a more conservative reaction to channel changes, where stronger codes are used more often. When threshold is decreased, the value PER converges to PER of the strongest code, since the strongest code is represented 
more often in the adaptive scheme. As shown in Table III, both switching strategies $U_{2} D_{1}$ and $U_{3} D_{1}$ can guarantee a PER below the given threshold. This does not hold for extremely low PER thresholds, which not even the static scheme with the strongest code is able to meet. The strategy $U_{1} D_{1}$ fails to keep PER below the threshold in all observed cases.

Compared to the strongest static FEC scheme considered, RS(15,5), AFEC always falls short in terms of PDR, because adaptive schemes use $\operatorname{RS}(15,5)$ only for a fraction of the time. It is not possible to reach the PDR of RS(15,5) unless AFEC permanently remains in the corresponding Markov state. However, there exists a trade-off and AFEC has two advantages over static coding with the strongest code. All three AFEC strategies provide higher throughput than the strongest static FEC scheme and require less transmissions, in all cases observed. For moderately high switching thresholds $\left(P E R_{T}=0.15\right.$ and 0.25 in Table III), strategies $U_{2} D_{1}$ and $U_{3} D_{1}$ also provide PER below the switching threshold.

The throughput advantage over $\mathrm{RS}(15,5)$ is more emphasized for higher switching threshold, because codes with higher code rate are forced. In case of $P E R_{T}=0.25$, this gain ranges between $66 \%$ and $107 \%$, depending on the switching strategy. For an extremely low switching threshold $\left(P E R_{T}=\right.$ 0 in Table III), all three strategies approach the reliability of RS(15,5), while still maintaining higher throughput.

Reduced energy consumption is accomplished due to a reduced number of necessary transmissions, compared to the codes with higher reliability (which simultaneously provide lower throughput). The rightmost column of Table III shows the number of transmissions required to deliver 90,000 bytes. The necessary number of transmissions is more than halved for $P E R_{T}=0.25$, compared to $\mathrm{RS}(15,5)$ case. Having in mind that the power-up and radio energy consumption are an important item in the sensor energy expenditure, substantial energy savings due to the reduced number of transmissions can be achieved.

The experimental results indicate AFEC algorithm is advantageous for applications that can tolerate a certain amount of packet loss. The gain for fault-tolerant applications is reflected in higher throughput and energy savings. In applications where high PDR is of uttermost importance compared to other performance parameters, the static FEC scheme with the strongest available code is the most appropriate solution. However, in that case, it is not possible to alter the coding and make use of good channel conditions in order to increase the throughput.

\section{CONCLUSIONS AND Future Work}

In this work, an Adaptive Forward Error Correction (AFEC) coding strategy for best effort Wireless Sensor Networks is proposed and its performance is compared to a static FEC coding scheme. Switching mechanism is based on recent history of transmission reliability, evaluated by monitoring the number of received ACKs within a time-window. Compared with common practice in adaptive FEC, our solution does not require additional feedback channels, which makes it compatible with networks that are unable to provide it. Furthermore, the scheme does not depend on rapidly changing RSSI values or network topology. Three switching strategies are assessed, out of which $U_{3} D_{1}$ provides the best reliability. Strategy $U_{1} D_{1}$ provides the highest throughput, but does not meet the PDR requirement in any of the cases examined. The simulation results show that AFEC cannot provide the PDR as high as the strongest static FEC scheme considered. The underlying cause is that AFEC blends codes of different powers and maintains the PDR above a predefined lower bound, which makes it more suitable for applications with a certain tolerance to packet loss. In these applications, AFEC can provide higher throughput and reduced energy consumption, compared to static FEC. However, static FEC scheme is a more desirable approach for networks with minimum or no tolerance for packet loss.

This work is the first step towards Adaptive Forward Error Correction for Best Effort Wireless Sensor Networks, rather than a self-contained solution. The next stage should include benchmarking with State-Of-The-Art RSSI- and SNR-based approaches, in order to justify the choice of link quality metric for this work and gain a deeper understanding of the contribution. A comparison with the performance of uncoded systems shall be undertaken. Finally, the proposed concept should be evaluated on error traces collected from actual field measurements.

\section{REFERENCES}

[1] J. Åkerberg, M. Gidlund, F. Reichenbach, and M. Björkman, "Measurements on an industrial wireless hart network supporting profisafe: A case study," in IEEE Conference on Emerging Technologies and Factory Automation (ETFA'11), Sept. 2011.

[2] A. Willig, "Recent and emerging topics in wireless industrial communications: A selection," Industrial Informatics, IEEE Transactions on, vol. 4, no. 2, pp. $102-124$, may 2008 .

[3] Z. Liankuan, X. Deqin, T. Yi, and Z. Yang, "Adaptive error control in wireless sensor networks," in Wireless Sensor Network, 2010. IET-WSN. IET International Conference on, nov. 2010, pp. $360-366$

[4] O. Eriksson, E. Björnemo, A. Ahlen, and M. Gidlund, "On hybrid arq adaptive forward error correction in wireless sensor networks," in The 37th Annual Conference of the IEEE Industrial Electronics Society(IECON), Nov. 2011.

[5] Y. Charfi, N. Wakamiya, and M. Murata, "Adaptive and reliable multipath transmission in wireless sensor networks using forward error correction and feedback," in Wireless Communications and Networking Conference, 2007.WCNC 2007. IEEE, march 2007, pp. 3681 -3686.

[6] C. Yan-ming, X. Yong-jun, W. Qiu-guang, and X. Lei, "An adaptive fault-tolerant scheme for wireless sensor networks," in Communications and Mobile Computing, 2009. CMC '09. WRI International Conference on, vol. 2, jan. 2009, pp. $32-36$.

[7] K. Yu, J. Åkerberg, M. Gidlund, and M. Björkman, "Reliable and low latency transmission in industrial wireless sensor networks," in The First International Workshop on Wireless Networked Control Systems (WNCS), sept. 2011, pp. 1-6.

[8] J. Proakis and M. Salehi, Digital communications, 5th ed. McGrawHill, Sept. 2007.

[9] "Ieee 802.15.4 specification," pp. 1 -305, 2006.

[10] Scalable network technologies, http://www.scalable-networks.com.

[11] W. Lee and D. Lee, "Microcell prediction in dense urban area," Vehicular Technology, IEEE Transactions on, vol. 47, no. 1, pp. $246-253$, feb 1998.

[12] A. Iqbal and S. A. Khayam, "Improving WSN Simulation and Analysis Accuracy Using Two-Tier Channel Models," in 2008 IEEE International Conference on Communications. IEEE, May 2008, pp. 349-353. [Online]. Available: http://dx.doi.org/10.1109/ICC.2008.72

[13] C. T. E. Jaein Jeong, "Forward error correction in sensor networks," May 2003 